\title{
Síndrome postlaminectomía lumbar I. Tratamiento del dolor mediante técnicas intervencionistas
}

\author{
F.J. Robaina Padrón
}

Unidad del Dolor Crónico y Neurocirugía Funcional Hospital Universitario de Gran Canaria “Dr. Negrín”

\section{Resumen}

La lumbalgia y la ciática postquirúrgicas representan unos síntomas por los que un elevado número de pacientes son remitidos a las Unidades del Dolor (UDO) en busca de soluciones a sus problemas. La futuras posibilidades de expansión de las Unidades Multidisciplinarias del Dolor (UMD) hacen que los neurocirujanos volvamos a recuperar el protagonismo perdido en los últimos años, por lo que es preciso exponer cuales son las técnicas que desde nuestra especialidad podemos aportar a las nuevas UMD. No se entra en los aspectos quirúrgicos del síndrome postlaminectomía lumbar, que ocasionalmente los tiene, sino en la descripción de las técnicas percutáneas intervencionistas. La utilización escalonada de los bloqueos vertebrales con control radiológico junto a las técnicas de radiofrecuencia, permiten abrir un gran abanico de posibilidades para el control de este tipo de dolor. Las técnicas de neuromodulación aplicables al manejo del dolor crónico, serán tratadas en otro artículo. Las técnicas neuroablativas a nivel medular y/o cerebral ya no tienen aplicación en esta patología. No obstante, la simpatectomía lumbar y la termoneurolisis, vuelven a cobrar importancia en el manejo del dolor crónico lumbociático postquirúrgico. Se describen los distintos algoritmos de manejo del dolor según la estructura afectada. El empleo de la radiofrecuencia pulsada permite abordar con seguridad el ganglio de la raíz dorsal e incluso el propio nervio raquídeo, disminuyendo las eventuales complicaciones de las antiguas técnicas de radiofrecuencia. Se describen en detalle los aspectos fundamentales para la realización de las diferentes técnicas intervencionistas, aportando imágenes reales, lo que permitirá asimilar más fácilmente el conocimiento que quiere transmitirse.

PALABRAS CLAVE: Síndrome postlaminectomía lumbar. Alivio del dolor. Técnicas intervencionistas. Bloqueos espinales. Radiofrecuencia.
Lumbar post-laminectomy syndrome I. Pain management using interventionist techniques

Summary

Chronic low back pain and ciatica after surgery is one of the main reasons for referring patients to multidisciplinary pain units (MPU). The future expansions of MPU have promted neurosurgeons in Spain to re-enter the field of chronic pain management and to recover their enthusiam, that have been lost severel years ago for various reasons. We do not intend dealing with the new instrumented surgical options for the post-laminectoy pain, but rather into the indications and descriptions of the interventional techniques for lumbar and sciatic pain managemet. Currently there are a great number of percutaneous techniques to achieve pain control in the post-laminectomy syndrome. Traditional neuroablative techniques at the spinal cord or the cerebral levels are not any longer indicated. Nonetheless, lumbar sympathectomy and thermo-neurolisis either with chilling or heating, are back in favour, due to the development of new devices and applications. Neuromodulation techniques which are also applicable to this pathology will be the subjet of another article. Pulsed radio-frecuency apperars to favour the safe application of electricity to the dorsal root ganglion or to the root itself thus avoiding the fear of permanent root and/or ganglion thermal lesions that frecuently occurred with the older techniques. Several strategies for pain relief are described, step by step and real figures are depicted in order to make the procedures more understandable and for the easier transmission of knowledge.

Abreviaturas. MPU: Multidisciplinaria Pain Unit. RFP: Radiofrecuencia Pulsada. RNM: Resonancia Nuclear Magnética SED: Sociedad Española del Dolor. SENEC: Sociedad Española de Neurocirugía. TAC: Tomografía Axial Computarizada. UDO: Unidad del dolor. UMD: Unidad Multidisciplinaria del dolor. UMTDC: Unidades Multidisciplinarias de Tratamiento del Dolor Crónico 
KEY WORDS: Lumbar post-laminectomy syndrome. Pain relief. Interventional techniques. Spinal blocks. Radiofrequency.

\section{Introducción}

El síndrome de fracaso de la cirugía lumbar, denominado también como síndrome post-laminectomía lumbar, no ha sido definido en concreto por ningún autor, ya que sus manifestaciones son conocidas por todos aquéllos que se dedican al manejo quirúrgico y postquirúrgico de la patología lumbar. Podríamos definirlo como el dolor crónico, persistente o recurrente que se presenta después de uno o más procedimientos quirúrgicos sobre la columna lumbosacra. Puede incluir una dorsalgia baja lo que tiene repercusiones a la hora de los tratamientos como ya veremos.

Los pacientes diagnosticados de ciática y lumbalgia postquirúrgica representan un número muy elevado de casos remitidos a las Unidades del Dolor (UDO) en busca de soluciones. Actualmente, existen diferentes modalidades de manejo de este tipo de dolor crónico, por lo que es preciso intentar su unificación y escalonamiento progresivo para no demorar la aplicación de los mismos. Es deseable que en el futuro inmediato se establezca una colaboración directa entre los neurocirujanos y ortopedas con perfil de dedicación a la patología quirúrgica de la columna vertebral, tanto a la hora de plantear las estrategias de tratamiento quirúrgico, como a la hora de manejar el dolor derivado de algunas de las técnicas quirúrgicas utilizadas.

Las tendencias actuales en nuestro país se dirigen rápidamente hacia la liberalización de las UDO de los Servicios de Anestesia y Reanimación, la creación de un área de capacitación especial en manejo del dolor crónico, solicitud ésta ya presentada en el Ministerio de Sanidad en el año 2004 firmada entre otras por la Sociedad Española de Neurocirugía (SENEC) y la Sociedad Española del Dolor (SED). Esta confluencia de objetivos permite ser optimistas en relación "al regreso de los neurocirujanos" con pleno derecho a participar y poder dirigir si fuera preciso, las actividades de las futuras Unidades Multidisciplinarias de Tratamiento del Dolor Crónico (UMTDC).

La revitalización de las técnicas clásicas de radiofrecuencia, el desarrollo de nuevas aplicaciones de la misma, el gran avance de las técnicas de neuromodulación, así como las nuevas aplicaciones de la cirugía mínimamente invasiva para el abordaje quirúrgico de la columna vertebral, hacen necesario la unificación de los criterios de manejo en estos pacientes.

Las técnicas de diagnóstico por imagen, si no son interpretadas correctamente en función de la clínica de los pacientes, pueden dar lugar en muchas ocasiones a confusión y derivar en la aplicación de técnicas intervencionistas no indicadas.

El origen de los pacientes que actualmente se atiende en las UMTDC es muy variable, aunque son los Servicios de Neurocirugía, Unidades de Cirugía del Raquis, Rehabilitación y Reumatología, los que remiten con mayor frecuencia. No es infrecuente que el médico de atención primaria, cansado de ver a su paciente sin una orientación adecuada, lo remita directamente a las UDO.

\section{Tipos de dolor en el síndrome postlaminectomía lumbar}

Básicamente, debemos distinguir dos tipos de dolor en estos síndromes álgicos de la columna vertebral intervenida quirúrgicamente. Uno el lumbar, generalmente de características somáticas y mecánicas y el otro neuropático, localizado en las extremidades inferiores. Es posible que el primero posea también en ocasiones aspectos neuropáticos, ya que la forma en que practicamos actualmente la mayor parte de la cirugía de la columna ocasiona denervaciones importantes de la musculatura paravertebral de la zona intervenida.

El dolor lumbar es fundamentalmente musculoesquelético, con un patrón de irradiación generalmente no metamérico, pudiendo aumentar por las noches. Es mecánico, agravándose con las flexiones, extensiones y rotaciones de la columna. El dolor neuropático predomina en las extremidades inferiores y se presenta frecuentemente de forma insidiosa, con un patrón de irradiación metamérico. Los pacientes con dolor neuropático suelen referir sensaciones alodínicas (alodinia: sensación desagradable provocada por un estímulo no nociceptivo) en la extremidad, objetivándose además con mucha frecuencia disminución de temperatura en la extremidad, tanto subjetiva como objetivamente. Existen en estos cuadros neuropáticos paroxismos de dolor y, en ocasiones, pueden aparecer cambios tróficos y vasomotores en la extremidad afecta.

La historia clínica y las características generales del dolor nos definirán, por lo tanto, si estamos ante un dolor de causa somática por exceso de nocicepción, un dolor neuropático de predominio distal fundamentalmente, o bien si el cuadro es mixto. También podremos valorar la posible influencia del Sistema Nervioso Simpático en estos síndromes dolorosos según se manifiesten en mayor menor medida las alteraciones vasomotoras y termoreguladoras en las extremidades inferiores.

\section{Escalonamiento terapéutico}

La estrategia terapéutica en estos casos debe ser escalonada y progresiva en cuanto a complejidad, continuándose en el tiempo sin interrupciones, ya que los pacientes han sido sometidos previamente a diferentes modalidades de tratamiento farmacológico y/o rehabilitador, generalmente 
con escaso éxito. Es necesario por lo tanto iniciar un protocolo de actuaciones tendentes a la máxima efectividad con la menor morbilidad ${ }^{17}$.

Los especialistas en el manejo del dolor crónico lumbar deben tener nociones muy precisas de la anatomía de la región para poder identificar las posibles fuentes responsables de los distintos síndromes dolorosos. Además, los conocimientos anatómicos de la zona posibilitarán la realización de las diferentes técnicas intervencionistas con un elevado grado de fiabilidad. Así, en la región lumbar, debemos tener presente que las distintas estructuras que pueden originar dolor crónico o dar lugar a complicaciones al realizar las diferentes técnicas son: sistema nervioso simpático, ganglio de la raíz dorsal, raíces lumbosacras, ramos posteriores postprimarios, venas y arterias radiculares, plexos venosos perirradiculares y epidurales, grasa epidural, médula espinal (niveles altos), ligamentos vertebrales y los componentes óseos de cada segmento móvil ${ }^{7}$.

En general, las técnicas intervencionistas que deben aplicarse en este tipo de pacientes son ampliamente conocidas por los especialistas en el manejo del dolor crónico, pero su aplicación debe ser progresiva y escalonada, como ya se ha comentado, para evitar tanto demoras en el diagnóstico como retrasos en la necesidad de realizar nuevos procedimientos quirúrgicos.

Básicamente, los recursos diagnóstico-terapéuticos que deben aplicarse en este tipo de patología son:

1. Bloqueos facetarios: anestesia local y esteroides.

2. Bloqueos sacroiliacos.

3. Denervaciones facetarias con radiofrecuencia o criolesiones.

4. Bloqueos epidurales: anestésico local, esteroides, morfina. Ventrales, dorsales, técnicas de doble catéter ${ }^{7}$.

5. Bloqueos del ganglio de la raíz dorsal.

6. Bloqueos radiculares o foraminales selectivos.

7. Lisis de las adhesiones epidurales ${ }^{15}$.

Algunas de las técnicas anteriores son pronósticas y pueden dar paso a otro grupo de técnicas “algo más intervencionistas" como son:

8. Simpatectomía lumbar: química o con radiofrecuencia (a nivel del ramo comunicante o del ganglio simpático).

9. Radiofrecuencia intradiscal.

10. Radiofrecuencia del ganglio de la raíz dorsal.

11. Técnicas de neuromodulación: Estimulación medular e infusión espinal de medicamentos

12. Procedimientos neuroablativos.

13. Nueva cirugía de columna: por vía anterior, posterior y/o laparoscópica.

\section{Utilización práctica de las técnicas intervencionistas}

Ante un cuadro de dolor lumbar persistente, con o sin ciática asociada, deben aplicarse una batería de bloqueos vertebrales diagnósticos. Así, podemos realizar un bloqueo radicular selectivo; bloqueos de las facetas articulares; bloqueo sacro-iliaco ; discografía. Técnicamente deben realizarse bajo control radiológico (intensificador de imágenes o TAC) y en una zona aséptica para disminuir el riesgo de complicaciones.

Los bloqueos radiculares o foraminales, nos permitirán definir correctamente si el dolor es o no dependiente de una o varias raíces. Para la realización de los mismos, además del empleo de los rayos $\mathrm{x}$, es preciso seguir una técnica depurada, que evite la eventual transfixión de la raíz, lo que podría originar un cuadro de dolor mucho más intenso. Puede emplearse la técnica de doble aguja, la cual permite realizar la inyección en el propio agujero de conjunción Pueden emplearse distintas concentraciones y tipos de anestesia local, así como distintas medicaciones antinflamatorias, generalmente derivados esteroideos. Publicaciones recientes ponen de relieve el magnífico resultado que se puede obtener a nivel terapéutico con este tipo de bloqueo al evitar la cirugía de las hernias discales en porcentajes superiores al 75\% de los casos quirúrgicos, o permitir realizar un bloqueo pronóstico del resultado de la cirugía discal ${ }^{19,29}$.

La técnica del bloqueo foraminal se realiza en quirófano, con el paciente en decúbito prono, en condiciones estériles y con control radiológico en proyecciones anteroposterior y lateral. Una vez localizado el nivel, el rayo se oblicua hacia el lado derecho o izquierdo del paciente aproximadamente 20-30 grados hasta observar perfectamente el pedículo. Nuestra diana se encuentra en un punto imaginario que correspondería a las 6 horas en caso de que el pedículo fuera como un reloj. En este punto, la raíz ya ha salido inferiormente y es poco probable que podamos lesionarla. En la proyección lateral, la aguja debe encontrarse en la parte superior del agujero de conjunción. A nivel sacro, la raíz S1 se puede bloquear a través del primer agujero sacro. Una vez que la aguja está en su sitio, a continuación se inyectan 1-2 cc de una mezcla de contraste yodado y bupivacaína al 0,25\%. Una vez localizada radiológicamente la raíz, se inyectan 2 cc de una mezcla de 1 cc de metilprednisolona o triamcinolona de depósito (40 mgr) y 1 cc de bupivacaína al 0,25\% (Figura 1).

La discografía y la inyección intradiscal de anestésicos locales y/o sustancias antinflamatorias, nos permitirán conocer el estado del disco y el anillo fibroso, además de comprobar si se induce dolor tras la inyección y el comportamiento del contraste, ya que éste puede permanecer en el interior del núcleo pulposo o emigrar al espacio epidural ${ }^{7}$.

Una de las técnicas intervencionistas empleadas con mayor frecuencia en el manejo de esta patología en las Unidades del Dolor son los bloqueos epidurales. La técnica 

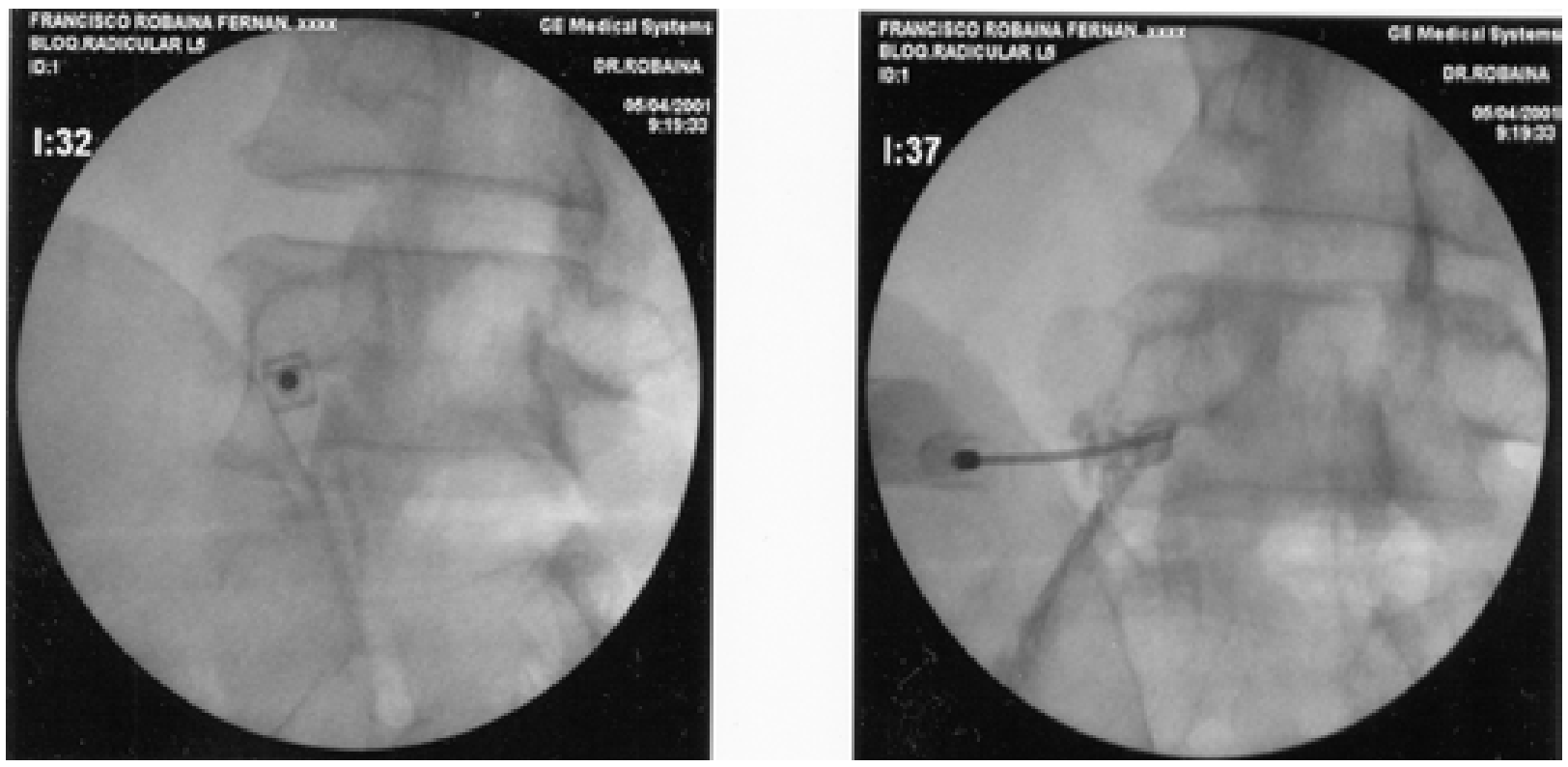

Figura 1. Izquierda: proyección AP en la que se observa cual es 1 target elegido a nivel L5-S1 izquerdo; en la zona inferior del pedículo de L5, aguja en visión túnel (eje del rayo y de la aguja coincidentes). Derecha: inyección de contraste que nos muestra el espacio perineural de la raíz L5. Las imágenes pertenecen al padre del autor que cuenta actualmente 79 años y que evitó hace 6 años una cirugía inminente por estenosis de canal y radiculopatía L5 izquierda incapacitante. Actualmente hace deporte 6 días a la semana y camina durante 5 horas (golf) de tres a cuatro veces en semana.

puede tener diferentes variantes. Desde la simple inyección epidural en sesiones semanales, pasando por la utilización de catéteres epidurales externalizados tipo DuPen, a los introducidos por el agujero caudal y por el agujero de conjunción ipsi o contralateral guiados con control radiológico y los conectados a reservorios o port-access subcutáneos?. Los fármacos utilizados son generalmente esteroides y anestésicos locales, aunque pueden asociarse pequeñas dosis de opiáceos, clonidina y orgoteína ${ }^{4}$.

Una variante muy interesante de los bloqueos epidurales es la emergente técnica de la lisis de las adhesiones epidurales (adhesiolisis) de las raíces tras la cirugía. Gabor Racz ha descrito y perfeccionado una técnica que consiste en la introducción de un catéter, especialmente diseñado al respecto, por el agujero caudal del sacro; asciende en el espacio epidural hasta posicionarlo lo más próximo posible a la raíz en cuestión, empleando para ello contraste radiológico y estimulación eléctrica. Posteriormente, procede a inyectar suero salino hipertónico con hialuronidasa para tratar de liberar la raíz. Esto no es siempre posible, ya que, en ocasiones, la cicatriz epidural es tan plástica que adhiere completamente la duramadre y la vaina dural radicular a las estructuras óseas del foramen y del cuerpo vertebral, tal y como se comprueba frecuentemente cuando se intenta una lisis quirúrgica. La base fisiopatológica en la que se apoya la técnica de la lisis epidural reside en la posibilidad, por un lado, de liberar físicamente la raíz de las adhesiones y, por otro lado, disminuir la congestión venosa y el edema radicular en la zona, aumentando el espacio disponible para la raíz en el agujero de conjunción ${ }^{15}$.

En relación a las técnicas neuroablativas, existen unas más invasivas que otras, por lo que su aplicación en el tratamiento del dolor lumbar y en la ciática persistente de origen no neoplásico están muy limitadas. Así, las técnicas de termoneurolisis (crioanalgesia y radiofrecuencia) se limitan en la práctica diaria a la realización de procedimientos intradiscales, denervaciones facetarias y termolesiones del ganglio de la raíz dorsal y, últimamente, al ramo comunicante simpático paravertebralmente. La crioanalgesia, por el calibre de los electrodos, está muy limitada y se aplica exclusivamente en las denervaciones del ramo posterior postprimario en el síndrome facetario ${ }^{13,10}$.

La simpatectomía lumbar con radiofrecuencia puede tener un cierto campo de aplicación siempre que se compruebe previamente mediante bloqueos diagnósticos que el dolor neuropático o discogénico que refiere el paciente posee un importante componente simpático ${ }^{20}$. En el apartado de las técnicas de radiofrecuencia se hará mención nuevamente a ésta técnica y se describirá la misma.

Las neurolisis periféricas, neurectomías y otras técnicas quirúrgicas como la cordotomía cervical percutánea, la mielotomía comisural o la lesión en la zona de entrada de las raíces posteriores en la médula (DREZ), no tienen ningún sentido en esta patología en ningún caso $^{18}$. 


\section{Técnicas de radiofrecuencia}

La producción de lesiones para destruir selectivamente algunas vías o núcleos del sistema nervioso central o periférico para modificar (actualmente se denomina "neuromodular”), se remonta a bastantes años atrás. Según Kline ${ }^{9}$, las ventajas de las técnicas de radiofrecuencia sobre otros métodos neurodestructivos son:

1. Se puede controlar el tamaño de la lesión.

2. Se puede controlar en todo momento la temperatura en el extremo del electrodo.

3. Se puede comprobar la localización del electrodo mediante el empleo de la impedancia, la frecuencia y el voltaje de la corriente eléctrica.

4. Los electrodos de radiofrecuencia pueden emplearse para diferentes modalidades de tratamiento.

5. Estas técnicas pueden realizarse bajo anestesia local y escasa sedación.

6. El procedimiento no es totalmente destructivo, existiendo recuperación de la función nerviosa.

7. Escasa morbilidad y mortalidad, si se realizan las técnicas apropiadamente.

8. Las lesiones pueden repetirse nuevamente si reaparece el fenómeno patológico inicial.

El primer equipo comercial de radiofrecuencia se construyó en los años 50, inicialmente para producir lesiones cerebrales. En la realización de un procedimiento de radiofrecuencia el cuerpo se convierte en un elemento del circuito eléctrico, donde el electrodo activo es el que produce la lesión y el electrodo indiferente o dispersivo es una superficie del cuerpo seleccionada al colocar un placa dispersiva sobre ella.

Uno de los aspectos importantes de la radiofrecuencia es que existe un rango de temperatura conocido como "zona de reversibilidad de las lesiones". Por ejemplo, una lesión cerebral entre 42,5 y $44^{\circ} \mathrm{C}$ es reversible, mientras que temperaturas superiores a $45^{\circ}$ crean una lesión tisular irreversible. En el sistema nervioso periférico, este aspecto del rango de la temperatura de los electrodos, permite aplicarlo a la destrucción selectiva de las fibras nociceptivas.

La monitorización de la temperatura en el extremo del electrodo activo es fundamental, no sólo para producir la lesión deseada, sino para evitar el sobrecalentamiento de los tejidos. Debe recordarse que el electrodo calienta al tejido y viceversa. El tiempo de duración de paso de la corriente así como la geometría del electrodo también son parámetros fundamentales. Para conseguir una lesión permanente la corriente debe estar pasando durante 30 a 60 segundos como mínimo ${ }^{11}$. La cercanía a vasos sanguíneos, líquido cefalorraquídeo o hueso, puede alterar la cantidad de calor generado en la punta del electrodo.

Los modernos equipos de radiofrecuencia deben contar con suficientes componentes electrónicos que faciliten el control y la lectura de los diferentes parámetros eléctricos necesarios para producir y controlar la lesión.

\section{Aplicaciones clínicas de la técnicas de radiofrecuencia en los síndromes de dolor lumbociático postquirúrgico}

Debido al auge de las Unidades Multidisciplinarias del Dolor (UMD) integradas entre otros por neurocirujanos y anestesiólogos con especial interés en la patología de columna, cada vez son más los pacientes remitidos para estudio y tratamiento de diferentes síndromes dolorosos lumbociáticos. Los procedimientos de radiofrecuencia representan un arma terapéutica muy útil en las UMD si se emplean con juicio y ateniéndose a una técnica depurada. Es fundamental destacar una vez más la necesidad de contar con unos equipos de radiofrecuencia fiables, con unos equipos radiológicos con memoria y que además cuenten con la posibilidad de grabación en papel, video o disquete, con una doble finalidad, docente y medicolegal.

Las técnicas de radiofrecuencia se encuentran a lo largo del camino de la estrategia terapéutica. En el momento de su aplicación, las técnicas de localización, estimulación y lesión, la anatomía regional y radiológica, así como el funcionamiento de los equipos auxiliares de radiología, deben conocerse perfectamente antes de intentar cualquier tipo de lesión, por sencilla que se considere.

\section{Radiofrecuencia pulsada}

Un refinamiento de las técnicas clásicas de radiofrecuencia con más de 25 años de uso, lo constituye la radiofrecuencia pulsada (RFP). Consiste en la aplicación de una corriente eléctrica de un voltaje alto pero sin inducir la elevación de la temperatura que origina la lesión nerviosa (no superar los $42^{\circ} \mathrm{C}$ ). Su campo de aplicación es el dolor neuropático. Su mecanismo de acción se ha postulado a través de un fenómeno de bloqueo persistente de la transmisión nociceptiva a nivel medular ${ }^{12,21}$.

Los síndromes dolorosos de la columna lumbar y concretamente los de la unión lumbosacra, representan un volumen elevado de pacientes que son remitidos a las UMD. Es fundamental conocer perfectamente la anatomía de la zona, interpretar correctamente la sintomatología, la exploración neurológica y realizar diagnósticos diferenciales en función de los hallazgos clínicos y radiológicos.

\section{Tratamiento del dolor lumbar mediante radiofrecuencia}

El manejo del dolor lumbar crónico pre o postquirúrgico mediante técnicas de radiofrecuencia hace preciso seguir una metodología de tratamiento. La más conocida es la descrita por Sluijter en un libro de reciente edición ${ }^{22}$. Este autor propone inicialmente que si existe dolor sobre la L2, 


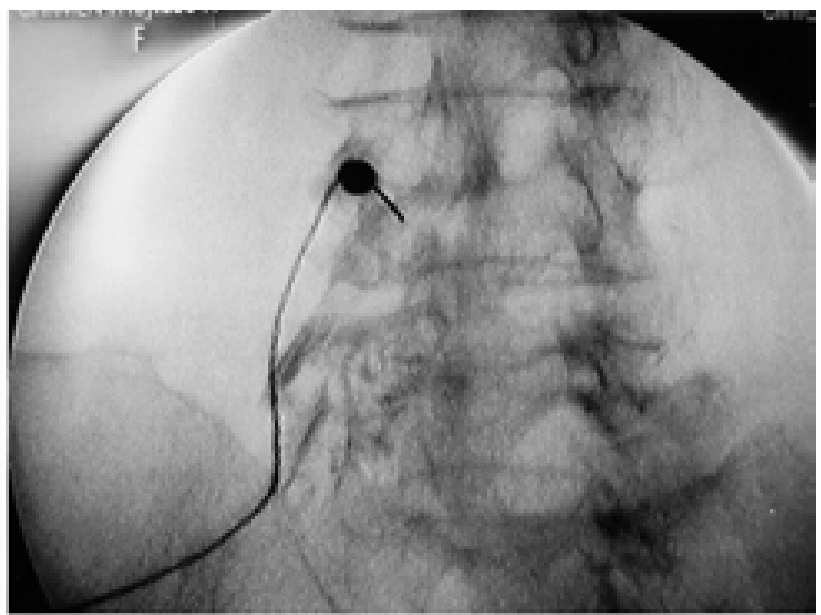

Figura 2. Radiofrecuencia pulsada de la raíz L4 izquierda después de su visualización con contraste hidrosoluble.

a nivel de la costilla, se practique inicialmente un bloqueo del nervio segmentario a ese nivel y si es positivo realizar entonces una RFP del ganglio de la raíz D12. Seguidamente, se plantea la realización de un bloqueo de la faceta articular o del ramo posterior y eventualmente un procedimiento de RF normal o RFP.

El dolor discogénico es en este momento el punto de atención. Su inervación como veremos más adelante es muy compleja. Muchos pacientes con dolor discogénico responden muy bien a un bloqueo del nervio raquídeo L2 por lo que en caso de que sea positivo una RFP de la raíz L2 puede conseguir un alivio persistente en el $50 \%$ de los casos. Si el bloqueo es negativo o el alivio no es persistente, el paso siguiente es la realización de un bloqueo simpático inmediatamente por encima del disco patológico y eventualmente una simpatectomía con RFP.

El paso siguiente en el dolor discogénico es el bloqueo de los nervios segmentarios por encima y por debajo del disco patológico. Cuando estos bloqueos son positivos una RFP del ganglio o de la propia raíz de estos nervios es el paso siguiente.

Los procedimientos intradiscales con radiofrecuencia son los únicos que pueden producir complicaciones graves por lo que deben dejarse para el final. Por otro, lado todavía no se conoce la eficacia de estos procedimientos, estando inicialmente contraindicados en pacientes ya operados.

\section{Tratamiento del dolor en la extremidad inferior me- diante radiofrecuencia}

El primer paso es la práctica de un bloqueo diagnóstico segmentario a nivel foraminal (Figura 1) seguido en caso de resultado positivo de un procedimiento con RFP sobre el ganglio de la raíz dorsal (Figura 2). Si esta estrategia resulta negativa, el paso siguiente sería realizar un bloqueo simpá-

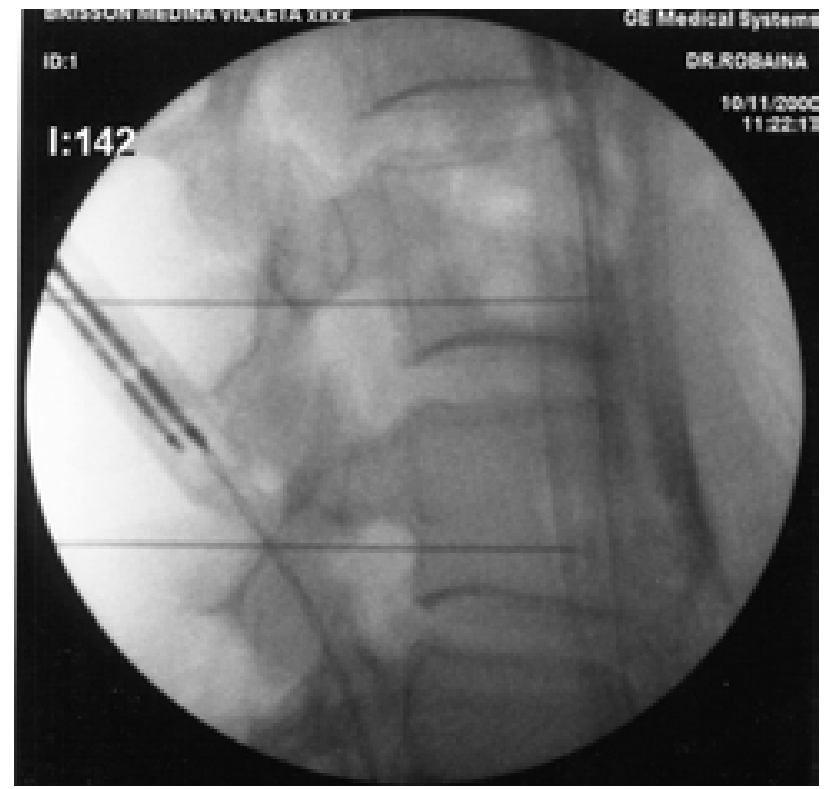

Figura 3. Simpatectomía lumbar con radiofrecuencia. Inyección de contraste hidrosoluble previamente para definir el borde antero-lateral de las vértebras L3-L4-L5.

tico lo más cerca posible del nivel patológico y, eventualmente, una simpatectomía con RFP de la cadena simpática (Figura 3).

Si existe una combinación de dolor lumbar y en la extremidad inferior, el orden de actuaciones estará en función de la intensidad del dolor en la pierna. Si el dolor sigue un trayecto metamérico, es mejor empezar por este nivel ya que un número importante de pacientes alivia de forma muy significativa. Curiosamente, en la experiencia de Sluijter, mejoran más los pacientes con afectación de L4 y S1 que los que tiene afectada la raíz L5. En caso de persistencia de dolor lumbar, éste debe tratarse de forma independiente pudiendo responder a un simple bloqueo de facetas o del propio ramo posterior.

En caso de que el dolor tenga un recorrido pseudorradicular o no se extienda por todo el dermatoma, debe aplicarse entonces radiofrecuencia a nivel lumbar, en las facetas articulares. En estos casos el dolor puede ser referido desde las facetas articulares de L4 y L5 o desde el disco por el dermatoma L2.

\section{Síndrome facetario postquirúrgico. Tratamiento con ra- diofrecuencia}

La faceta lumbar constituye una estructura que genera gran cantidad de cuadros de dolor crónico. El síndrome facetario fue descrito en 1933 por Ghormely ${ }^{8}$. Se calcula que entre el $50-67 \%$ de los pacientes que han sido seleccionados correctamente para un procedimiento de radiofrecuencia por este diagnóstico consiguen alivio del dolor ${ }^{1,6,14,16,23,26,27}$. 

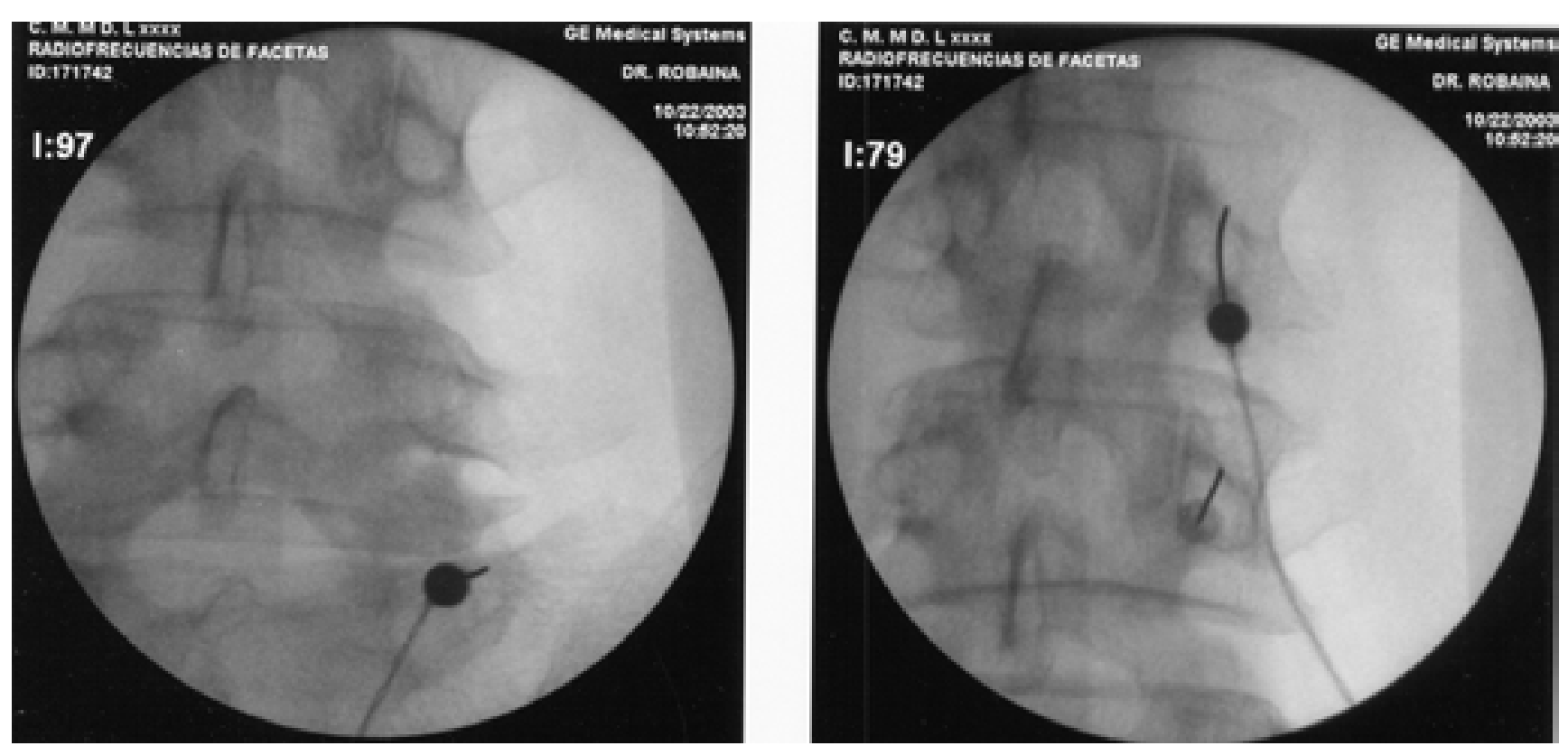

Figura 4. Radiofrecuencia de facetas lumbares. Obsérvese cómo el target se encuentra a nivel de las vértebras lumbares en el surco entre la apófisis articular ascendente y la transversa.

Los clínicos del dolor deben saber diagnosticar y reconocer cuando se enfrentan a un síndrome facetario o a un síndrome de dolor discogénico ${ }^{2,5}$. Éste ocurre en ausencia de herniación discal, incluso los estudios por imagen pueden aparecer normales (RNM y TAC). Solamente la sospecha clínica y su confirmación mediante maniobras de provocación, como la discografía, o de alivio del dolor por bloqueos anestésicos con o sin antinflamatorios, pueden inducirnos a realizar un procedimiento de radiofrecuencia en una u otra estructura.

El ramo posterior postprimario, rama del nervio raquídeo, se origina de éste tan pronto sale del agujero de conjunción. Se divide en dos ramas a medida que rodea al proceso articular superior de la vértebra inferior. La rama superior se dirige a inervar la articulación de su nivel y la otra desciende a la articulación inferior. Por lo tanto es en la unión entre la articular superior y la apófisis transversa donde debe realizarse el procedimiento. Para denervar un nivel hay que hacer también el nivel superior ${ }^{24}$.

Generalmente el equipo de rayos debe colocarse inicialmente en proyección anteroposterior, con una inclinación craneo-caudal que permita observar que los platillos vertebrales del nivel que vamos a tratar se encuentren paralelos. Posteriormente, se oblicua el arco radiológico hacia el lado donde vayamos a realizar el tratamiento hasta localizar el surco entre la apófisis articular ascendente y la apófisis transversa. Se marca este punto sobre la piel y se infiltra la piel y el plano muscular con anestesia local (lidocaína 1\%, 1-3 cc). Hay que intentar siempre la visión túnel para evitar desplazamientos de la punta de la aguja de radiofrecuencia.
Las agujas que se utilizan están especialmente diseñadas para estos procedimientos. Toda la aguja salvo la punta (2 ó $5 \mathrm{~mm}$ ) se encuentra aislada para el paso de la corriente. Generalmente con una aguja de $10 \mathrm{~cm}$ se alcanza perfectamente el target. (Figura 4).

Una vez en el punto seleccionado, se procede a estimular a 50 ciclos por segundo con una intensidad no superior a 0,5 voltios. La respuesta debe estar localizada en la región lumbar, generalmente en forma de una sensación de presión o dolor. La lesión puede hacerse mediante la técnica tradicional a $80^{\circ} \mathrm{C}$ durante 60 segundos o mediante radiofrecuencia pulsada.

Esta técnica selectiva es mucho más precisa que la inyección de anestésico y/o esteroide en la cápsula de la faceta articular. Después del procedimiento no existen restricciones para el paciente, pero se le debe advertir que el resultado del mismo no puede evaluarse correctamente hasta que pasen al menos dos semanas.

\section{Dolor discogénico. Tratamiento con radiofrecuencia}

Como ya se ha comentado anteriormente, en ocasiones, solamente los bloqueos anestésicos o la discografía provocadora de dolor pueden hacernos pensar que nos enfrentamos a un síndrome de esta naturaleza. Clínicamente predomina el dolor lumbar sobre la ciática, siendo más intenso durante las maniobras de flexoextensión, sobre todo durante la deflexión y al sentarse. Este tipo de pacientes significativamente experimentan una sensación extrema de fatiga o debilidad en las extremidades inferiores después 
de actividades físicas moderadas ${ }^{5}$.

La inervación del anillo fibroso del disco se realiza a través de los nervios sinuvertebrales provenientes a su vez del ramo anterior ventral de la raíz metamérica, de los ramos comunicantes grises y de ramas de la cadena simpática. Generalmente, las técnicas de radiofrecuencia discal se dirigen a generar una lesión en los ramos comunicantes grises y en el interior del disco. Estudios neuroanatómicos sugieren que los ramos comunicantes grises envían ramas a más de un nivel. Por este motivo, en dolor discogénico generado en el disco L4-L5 por ejemplo, deberían lesionarse los ramos comunicantes grises en L4 y en $\mathrm{L}^{24}$. El procedimiento tiene que ser bilateral. Los detalles de la técnica pueden estudiarse perfectamente en el libro de Menno E. Sluijter, dedicado exclusivamente al manejo con radiofrecuencia de la patología lumbosacra ${ }^{22}$.

Las técnicas de radiofrecuencia sobre el propio disco, tanto las dirigidas al núcleo denominadas nucleoplastia como las dirigidas al anillo denominadas anuloplastia, no son en principio aplicables a pacientes ya operados por lo que no se comentarán sus indicaciones y la técnica de aplicación ${ }^{3}$.

Antes de finalizar este apartado, siguiendo las indicaciones de Sluijter, hay que destacar que la diferencia entre un dolor de origen facetario y uno discogénico a veces no es posible, por lo que debe realizarse siempre un bloqueo facetario o del ramo posterior antes de plantear cualquier otro tipo de procedimiento sobre el disco o su inervación, ya que esto último implica mayor riesgo de complicaciones, como podría ser una discitis ${ }^{21}$.

Los procedimientos a nivel del ganglio de la raíz dorsal a nivel lumbar están indicados en el dolor persistente en la extremidad inferior. En general, este procedimiento está indicado en aquellas situaciones en las que se ha descartado cualquier otro tipo de procedimiento más eficaz y los bloqueos radiculares diagnóstico-terapéuticos han sido positivos temporalmente ${ }^{28}$. (Figura 2).

Es posible realizar también ganglionectomías con radiofrecuencia de las raíces sacras, pero la técnicas se complica al tener que perforar el sacro con una aguja de Kischner o un pequeño drill para poder acceder al ganglio, el cual se encuentra oculto en el interior del canal y no puede alcanzarse a través del correspondiente agujero sacro. La llegada de la RFP ha obviado este inconveniente ya que se puede actuar directamente sobre la raíz propiamente como es el caso de los nervios raquídeos dorsales y sacros ${ }^{21}$.

\section{Tratamiento con radiofrecuencia del dolor originado en la articulación sacroiliaca}

La articulación sacroiliaca es una de las más fuertes del organismo. Existen defensores y detractores sobre el tipo de dolor originado en esta articulación, dado que el dolor puede ser referido a esta zona desde las facetas articulares o desde el disco L2. Estos orígenes deben descartarse antes de proceder directamente sobre la articulación sacroiliaca. La inervación de la articulación es compleja pero suele responder al bloqueo de los ramos posteriores de las dos primeras raíces sacras en el borde de los respectivos agujeros de conjunción y que se dirigen lateralmente hacia la articulación. El nervio S2 puede someterse también a una RFP a través del correspondiente foramen.

En algunas ocasiones el dolor es puramente sacro con irradiación a las nalgas pero no más abajo. Este tipo de dolor se observa con alguna frecuencia en pacientes operados de columna lumbar con o sin artrodesis, pudiéndose intentar su tratamiento mediante múltiples procedimientos a nivel de las raíces sacras bilateralmente con RFP.

La articulación sacroiliacas es también responsable de dolor sacro bajo, de características mecánicas, irradiándose al pliegue inguinal, trocánter y cara anterior del muslo. Los bloqueos de la articulación pueden predecir el resultado de un procedimiento con radiofrecuencia ${ }^{1,27}$.

En muchas ocasiones es suficiente con realizar varias lesiones a $80^{\circ} \mathrm{C} 60$ segundos a lo largo de la articulación. Podría complementarse con la neurolisis con radiofrecuencia del ramo posterior de S1 y S2 a nivel del borde externo de primero y segundo agujeros sacros ya que éstos inervan también a dicha articulación.

En casos de coxigodinia, la realización de uno o varios procedimientos de RFP en el $4^{\circ}$ foramen sacro resulta en opinión de Sluijter altamente positivo si el paciente no ha sido operado a ese nivel.

\section{Dorsalgia postquirúrgica. Tratamiento con radiofre- cuencia}

La faceta dorsal puede ser también una fuente de dolor en el síndrome postlaminectomía lumbar tal y como observamos en la clínica diaria y ésta puede ser abordada mediante técnicas de radiofrecuencia. Existen una serie de aspectos neuroanatómicos que hacen que la técnica sea algo diferente a la de la región lumbar, fundamentalmente en lo que hace referencia al manejo del intensificador de imágenes, debido a la unión entre la apófisis transversa y su correspondiente costilla.

Igualmente, en la región dorsal, se pueden practicar ganglionectomías. La existencia de la pleura hace que la técnica de abordaje al agujero de conjunción sea diferente según el nivel. En los niveles bajos, la técnica puede ser similar a la región lumbar. En los niveles altos, es preferible realizar un agujero en la lámina, en el nivel superior del agujero de conjunción donde reside el ganglio raquídeo y todo el procedimiento controlado mediante una proyección lateral del intensificador de imágenes ${ }^{25}$. (Figura 5). 


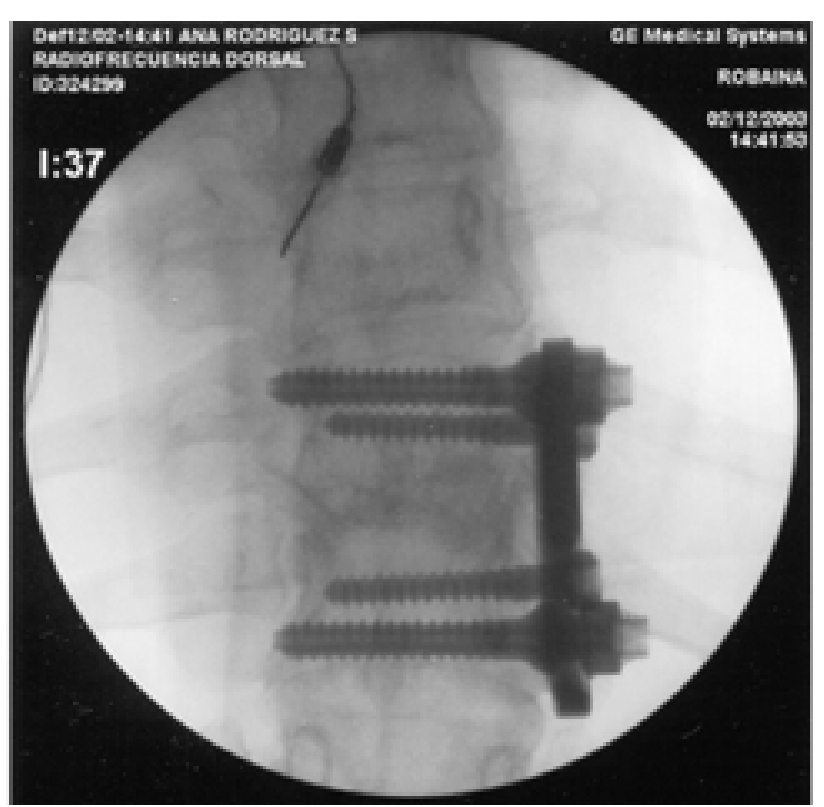

Figura 5. Radiofrecuencia de facetas dorsales en un caso de dolor postquirúrgico a nivel dorsal y lumbar. $\rightarrow$

\section{Radiofrecuencia de la cadena simpática en la región dorsal y lumbar en el síndrome postlaminectomía lumbar}

Es fundamental no realizar ningún tipo de lesión neurolítica sin previamente haber realizado bloqueos anestésicos. La cadena simpática tanto a nivel dorsal como lumbar se encuentra en la vecindad de estructuras vasculares (aorta, vena cava), nerviosas (nervios metaméricos, genitourinarios) y viscerales como los uréteres a nivel lumbar o la pleura a nivel dorsal.

Al igual que todas las técnicas de radiofrecuencia, el conocimiento de la anatomía radiológica es vital. Recientemente, se han desarrollado cánulas curvas (Racz-Finch) que facilitan en gran medida la realización de la técnica tanto a nivel dorsal como lumbar ${ }^{30}$.

\section{Conclusiones}

Como puede observarse después de leer el presente artículo, los neurocirujanos somos generadores y receptores de pacientes con dolor crónico. Por diferentes razones que pienso deberían analizarse en otros foros, la mayoría de los neurocirujanos formados en España en los últimos 15 años han tenido escaso o ningún contacto con las técnicas percutáneas para el alivio del dolor que, como vemos, si se aplican correctamente, pueden evitar nuevas cirugías (muchas lamentablemente vuelven a fallar), y mejorar la calidad de vida de un gran número de pacientes. Existe actualmente una gran expansión de la neurocirugía funcional en general, siendo el tratamiento del dolor crónico uno de los aspectos fundamentales de esa parte de la
2007; 18: 468-477
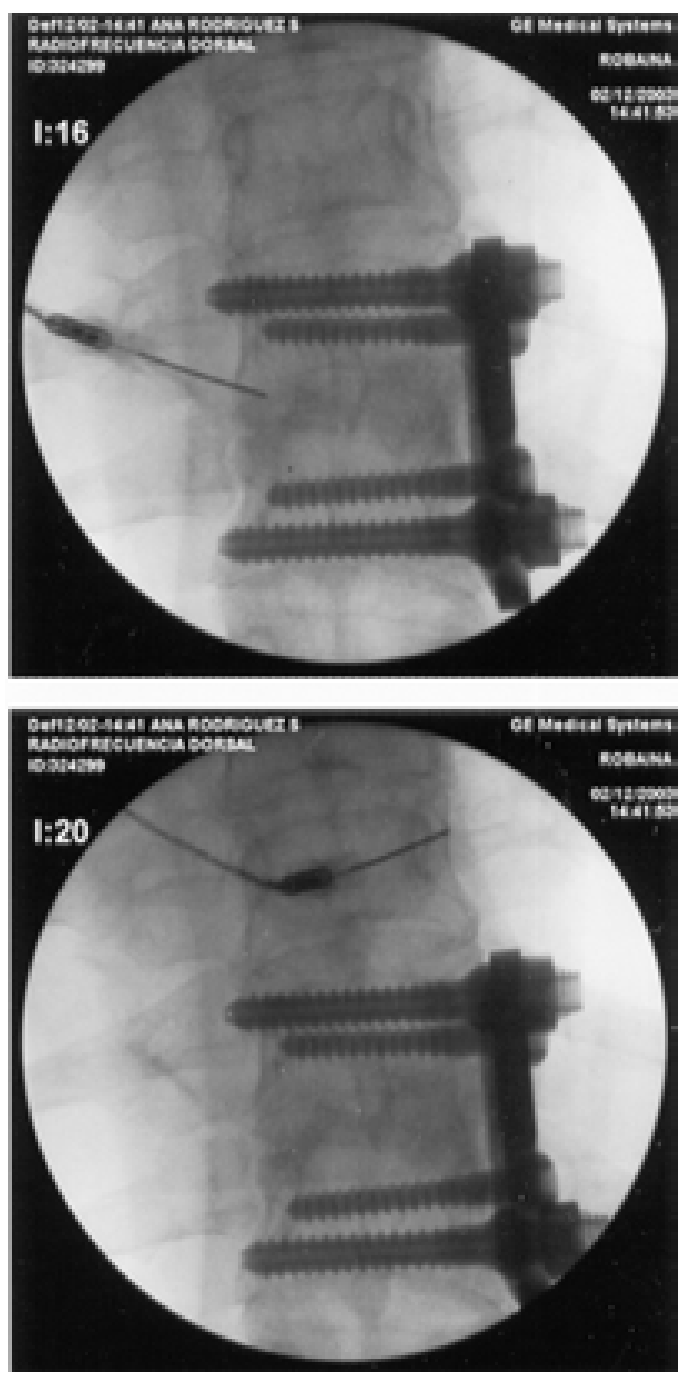

neurocirugía. El futuro a corto plazo es favorable para que los neurocirujanos jóvenes se incorporen a las UMTDC, donde pueden desarrollar una gran labor asistencial y donde además puede surgir una vía de incorporación a un puesto de trabajo estable.

Las técnicas de neuromodulación aplicables también al tratamiento del dolor lumbar y la ciática postquirúrgica serán objeto de un nuevo artículo.

\section{Bibliografía}

1. Anderson, K.H., Mosel, C., Varnet, K.: Percutaneous facet denervation in low back and extremity pain. Acta Neurochir 1987; 87: 48-51.

2. Aprill, C.: Diagnostic disc injection. Frymoyer JW (ed) The Adult Spine: Principles and Practice. Raven Press. New York, 1991; pp 403-442.

3. Barendse, G.A., van Den Berg, S.G., Kessels, A.H., Weber, W.E., van Kleef, M.: Randomized controlled trial of percutaneous intradiscal radiofrequency thermocoagulation 
for chronic discogénic back pain: lack of effect from a 90second 70 C lesion. Spine 2001; 26: 287-292.

4. Benzon, H.T.: Epidural steroids injections for low back pain and lumbosacral radiculopathy. Pain 1986; 24: 277-295.

5. Bernard, T.N.: Lumbar discography followed by computed tomography-refining the diagnosis of low back pain. Spine 1990; 15: 690-707.

6. Dreyfuss, P., Halbrook, B., Pauza, K., Joshi, A., McLarty, J., Bogduck, N.: Efficacy and validity of radiofrequency neurotomy for chronic lumbar zygapophysial joint pain. Spine 2000; 25: 1270-1277.

7. Finch, F., Taylor, R.: Functional Anatomy of the spine. En Waldman SD, Winnie AP (eds). Interventional Pain Management. Philadelphia ; Saunders Company 1996; pp. 39-68.

8. Ghormely, R.K.: Low back pain with special reference to articular facet with presentation of an operative procedure. JAMA 1933; 101: 1773-1777.

9. Kline, M.: Radiofrequency Techniques in Clinical Practice. En Waldman SD and Wennie AP (eds). Interventional Pain Management Philadelphia, W.B.Saunders 1996; pp.185217.

10. Kline, M.T.: Stereotactic Radiofrequency Lesions as Part of the Management of Pain. PMD, Florida, 1992.

11. Kline, M.T.: Radiofrequency Techniques in Clinical Practice. En Waldman SD , Winnie AP (eds) : Interventional Pain Management. Philadelphia. Saunders Company 1996; pp. 185- 217.

12 Munglani, R.: The longer term effect of pulsed radiofrequency for neuropathic pain. Pain 1999;80: 437-439.

13 North, R.B., Zahurak, M., Kidd, D.: Radiofrequency lumbar facet denervation: Analysis of prognostic factors. Pain 1984; 57: 77-83.

14 North, R.B., Kidd, D.: Radiofrequency lumbar facet denervation: Analysis of prognostic factors. Pain 1994; 57: 77 83.

15. Racz, G., Heavner, J.E., Diede, J.H.: Lysis of epidural adhesions utilizing the epidural approach. En Waldman SD, Winnie AP (eds). Interventional pain Management. Philadephia; Saunders Company, 1996; pp. 339-351.

16. Ray, C.D.: Percutaneous radiofrequency Facet Nerve Block Burlington: Radionics Procedure Technique Series. Radionics Corporation, 1982.

17 Robaina, F.: Manejo intervencionista de la ciática y del dolor lumbar postquirúrgico. Revista de la Sociedad Española del Dolor 1999; 6: 233-237.

18 Robaina-Padrón, F.J.: Tratamiento quirúrgico del dolor de espalda. Surgical treatment of back pain. Actualizaciones en Dolor 1, 2000; 1: 42-50.
19. Sasso, R., Macadaeg, K., Nordmann, D., Smith, M.: Selective nerve root injections can predict surgical outcome for lumbar and cervical radiculopathy. J Spinal Disord Tech 2005; 18: 471-478.

20. Sluijter, M.E.: The use of radiofrequency lesions for pain relief in failed back patients: International Disability Studies 1988; 10: 37-42.

21. Sluijter, M.E., Cosman, E.R., Rittman, W.B., Van Kleef, M.: The effects of pulsed radiofrequency fields applied to the dorsal root ganglion- a preliminary report. The Pain Clinic 1998;11: 109-117.

22. Sluijter, M.E.: Radiofrequency Part 1. A review of radiofrequency procedures in the lumbar region. FlivoPress SA. 2001.

23. Sluijter, M.: Radiofrecuency procedures for back pain and sciatica. Abstract Book. $4^{\text {th }}$ International Congress. International Neuromodulation Society. Lucerne, Switzerland. September. 1998.

24. Sluijter, M.E.: The role of radiofrequency in failed back surgery patients. Curr Rev Pain 2000; 4: 49-53.

25. Stolker, R.J., Vervest, A.C., Groen, G.J.: The treatment of chronic thoracic segmental pain by radiofrequency percutaneous partial rhizotomy. J Neurosurg 1994; 80: 986-993.

26. Tzaan, W.C.; Tasker, R.D.: Percutaneous radiofrequency facet rhizotomy-experience with 118 procedures and reappraisal of its value. Pain 2000; 84: 113-114.

27. Van Kleef, M., Barendse, G.A., Kessels, A., Voets, H.M., Weber, W.E., de Lange, S.: Randomized trial of radiofrequency lumbar facet denervation for chronic low back pain. Spine 1999; 24: 1937-1942.

28. Van Wuk, R.M., Geurts, J.W., Wynne, H.J.: Longlasting analgesic effect of radiofrequency treatment of the lumbosacral dorsal root ganglion. J Neurosurg 2001; 94: 227-231.

29. Wang, J.C., y cols.: Epidural injections for the treatment of syntomatic lumbar herniated discs. J Spinal Disord Tech 2002; 15: 269-272.

30. Wilkinson, H.A.: Percutaneous radiofrequency upper thoracic sympathectomy. Neurosurgery 1996; 38: 715-725.

Robaina Padrón, F.J.: Síndrome postlaminectomía lumbar I. Tratamiento del dolor mediante técnicas intervencionistas. Neurocirugía 2007; 18: 468-477.

Correspondencia postal: Dr. Francisco Javier Robaina Padrón. Jefe de la Unidad del Dolor Crónico y Neurocirugía Funcional. Nivel 5. Hospital Universitario de Gran Canaria Dr. Negrín. C) Barranco de la Ballena s/n. 35010 Las Palmas de Gran Canaria 